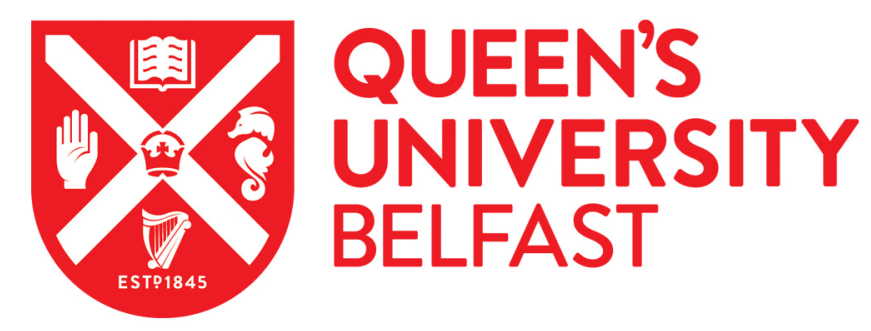

\title{
Angularly resolved characterization of ion beams from laser-ultrathin foil interactions
}

Scullion, C., Doria, D., Romagnani, L., Ahmed, H., Alejo, A., Ettlinger, O. C., Gray, R. J., Green, J., Hicks, G. S., Jung, D., Naughton, K., Padda, H., Poder, K., Scott, G. G., Symes, D. R., Kar, S., McKenna, P., Najmudin, Z., Neely, D., ... Borghesi, M. (2016). Angularly resolved characterization of ion beams from laser-ultrathin foil interactions. Journal of Instrumentation, 11, [C09020 ]. https://doi.org/10.1088/1748-0221/11/09/C09020

Published in:

Journal of Instrumentation

Document Version:

Peer reviewed version

Queen's University Belfast - Research Portal:

Link to publication record in Queen's University Belfast Research Portal

Publisher rights

Copyright 2016 IOP Publishing Ltd and Sissa Medialab srl

This work is made available online in accordance with the publisher's policies.

\section{General rights}

Copyright for the publications made accessible via the Queen's University Belfast Research Portal is retained by the author(s) and / or other copyright owners and it is a condition of accessing these publications that users recognise and abide by the legal requirements associated with these rights.

Take down policy

The Research Portal is Queen's institutional repository that provides access to Queen's research output. Every effort has been made to ensure that content in the Research Portal does not infringe any person's rights, or applicable UK laws. If you discover content in the Research Portal that you believe breaches copyright or violates any law, please contact openaccess@qub.ac.uk. 


\title{
Angularly resolved characterization of ion beams from laser-ultrathin foil interactions
}

\author{
C. Scullion ${ }^{a}$ D. Doria ${ }^{a}$ L. Romagnani ${ }^{b}$ H. Ahmed ${ }^{a}$ A. Alejo ${ }^{a}$ O.C. Ettlinger ${ }^{c}$ R.J.

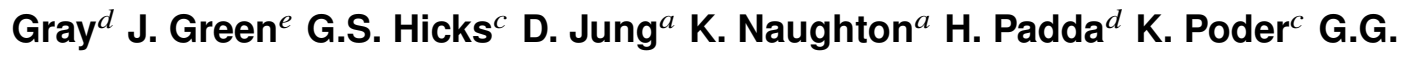

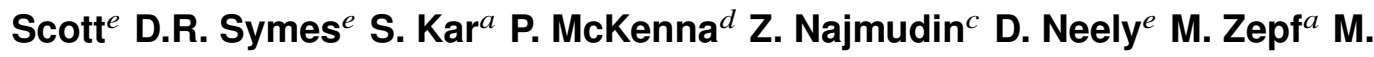 \\ Borghesi $^{a, 1}$ \\ ${ }^{a}$ Centre for Plasma Physics, School of Mathematics and Physics, Queen's University of Belfast, Belfast \\ BT7 INN, United Kingdom \\ ${ }^{b}$ LULI, Ecole Polytechnique, CNRS, Route de Saclay, 91128 Palaiseau Cedex, France \\ ${ }^{c}$ The John Adams Institute for Accelerator Science, The Blackett Laboratory, Imperial College, London \\ SW7 2BW, United Kingdom \\ ${ }^{d}$ SUPA, Department of Physics, University of Strathclyde, Glasgow G4 ONG, United Kingdom \\ ${ }^{e}$ Central Laser Facility, Rutherford Appleton Laboratory, Oxfordshire OX11 OQX, United Kingdom \\ E-mail: m.borghesi@qub.ac.uk
}

\begin{abstract}
AвSTRACT: Methods and techniques used to capture and analyze beam profiles produced from the interaction of intense, ultrashort laser pulses and ultrathin foil targets using stacks of Radiochromic Film (RCF) and Columbia Resin \#39 (CR-39) are presented. The identification of structure in the beam is particularly important in this regime, as it may be indicative of the dominance of specific acceleration mechanisms. Additionally, RCF can be used to deconvolve proton spectra with coarse energy resolution while mantaining angular information across the whole beam.
\end{abstract}

${ }^{1}$ Corresponding author. 


\section{Contents}

1 Introduction 1

2 Detectors and Processing Techniques 2

2.1 Radiochromic Film 2

2.2 CR-39 3

3 Application to Experiments 4

3.1 RCF 4

3.2 CR-39 5

3.3 RCF and CR-39 layers 5

4 Conclusions 6

\section{Introduction}

Laser driven ion acceleration is an area of research which has received significant interest [1] in recent years in view of the continuous advances in high power laser technology and of the possible applications in a range of areas including medical treatments [2] and nuclear fusion [3].

In addition to the well established Target Normal Sheath Acceleration (TNSA) mechanism [4, 5], the investigation of potentially more efficient processes such as Radiation Pressure Acceleration (RPA) and transparency enhanced acceleration (also referred to as Break Out Afterburner (BOA)) have motivated an intense experimental activity employing ultrathin foils (with thickness down to the $\mathrm{nm}$ range). Investigating these regimes in interactions employing ultrashort laser pulses (10's of fs) is of particular interest [6-8], as the relevant Ti:Sapphire (Ti:Sa) systems are inherently more compact and suitable for operations at high repetition, which are important features in view of potential applications. The ion beams in such experiments are primarily diagnosed using Thomson Parabola Spectrometers (TPS) coupled to image plate (IP) or micro-channel plate (MCP) detectors, providing energy-resolved ion spectra for different ion species [9-11]. However, measurements of the beam profile are also very important, as specific spatial/angular characteristics may be indicative of different acceleration regimes, and may help in isolating the dominant mechanism. For example, regular transverse modulations are characteristic of an unstable radiation pressure drive [12-14], while features associated to relativistic transparency regimes include beam elongation along the laser polarization direction [15], ring structures in the beam [16], as well as angularly separated 'double' beams for oblique incidence $[17,18]$.

Here, we present an overview of methods and techniques we used in recent campaigns aimed to characterize ion beams generated from the interaction of ultrashort, high-power laser pulses with ultrathin solid targets, with particular attention to the characterization of the beam profile. While 

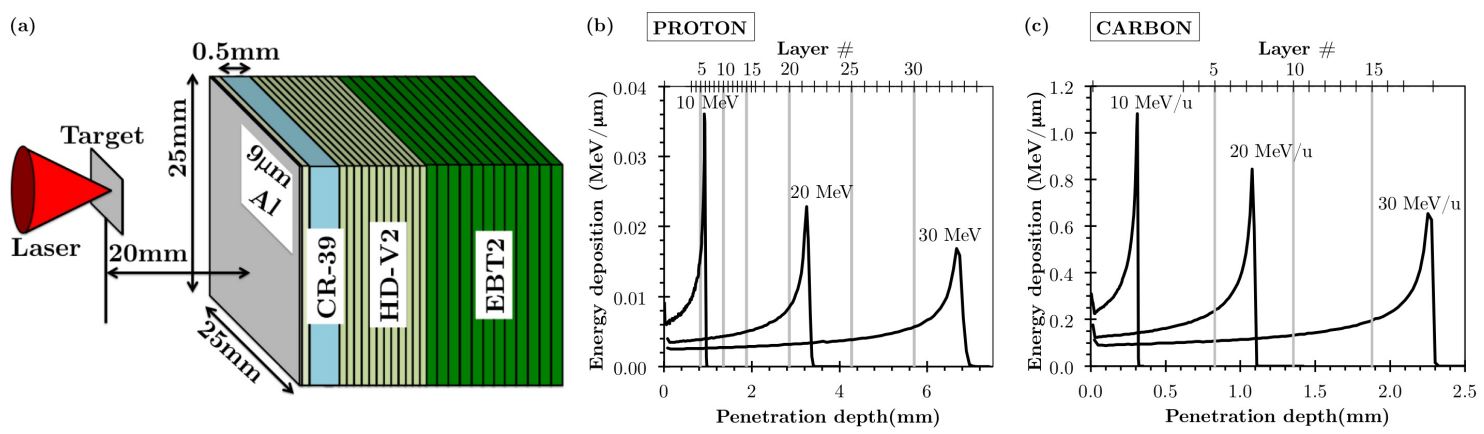

Figure 1. (a) An example of a stack configuration used in the experiment. From left to right the stack composition is $\sim 10 \mu \mathrm{m} \mathrm{Al}, 1 \times \mathrm{HD}-\mathrm{V} 2,500 \mu \mathrm{m}$ CR-39, $15 \times \mathrm{HD}-\mathrm{V} 2,30 \times$ EBT2. (b) Proton and (c) carbon stopping curves for the stack shown in (a).

the techniques presented are well established and generally applied over a broad range of interaction conditions (see [1] and references within), we will discuss how these are implemented in experiments investigating the interaction of intense, ultrashort pulses with ultrathin foils, and highlight the diagnostic requirements set by some specific aspects of the ion beams accelerated under these conditions, namely the structured nature of the beams and their multispecies composition where protons and higher- $Z$ species can reach comparable energies per nucleon.

We refer in particular to measurements carried out on the Astra GEMINI Ti:Sa laser system at the Rutherford Appleton Laboratory, STFC, United Kingdom. The laser of wavelength $\lambda, 800$ $\mathrm{nm}$, delivered 6-7 J energy on target within a pulse of $45 \mathrm{fs}$ full width at half maximum (FWHM) duration $\tau$, after being reflected off a double plasma mirror [19]. The laser was focused on the target at normal incidence by an $f / 2$ off-axis parabolic mirror, reaching peak intensity up to $3 \times 10^{20}$ $\mathrm{Wcm}^{-2}$. Amorphous carbon targets of thickness 2.5-100 nm were irradiated and the main species accelerated were protons and carbon ions. The energy spectra of the ions from the interaction were diagnosed by TPSs coupled to micro-channel plate (MCP) detectors, typically with an acceptance angle of $\sim 100 \mathrm{nsr}$. The spatial profile of the ion beam, with coarse energy resolution, was recorded with stacks of RCF and CR-39.

\section{Detectors and Processing Techniques}

\subsection{Radiochromic Film}

Gafchromic Radiochromic Film (RCF) is a self developing film which measures the dose deposited within its 'active layer', embedded in clear plastic. The optical density of the active layer instantly changes upon exposure to ionizing radiation and needs no chemical or physical treatment for development [20]. RCF stacks are commonly used as a diagnostic in high power laser experimental campaigns since they can provide information on the energy, transverse profile and structure of the accelerated ion beams [21-24]. In the experiments presented in this article HD-V2 [25] and EBT2 $[26,27]$ types were used as their different sensitivity allows to diagnose the beam over the large differences in particle density present across the spectrum.

Within a stack of RCF such as in Figure 1 (a), since ions deposit a fraction of their energy in all the RCF layers they penetrate through before being stopped, each RCF layer in the stack can be 


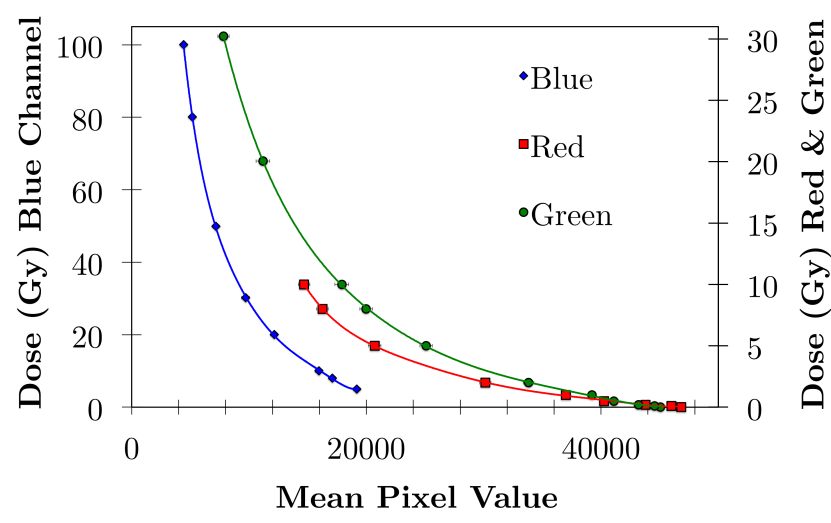

Figure 2. The relationship between dose (Gy) and pixel value in the red, green and blue colour channels for single EBT2 layers irradiated with known doses at the Birmingham cyclotron and scanned with an Epson Perfection V750 Pro optical scanner.

correlated to a particular proton energy range, whose lower limit is defined by the energy of protons whose Bragg peak occurs in its active layer. The proton and carbon energy loss and penetration depth were calculated using a Monte Carlo code SRIM (Stopping and Range of Ions in Matter) [28] and are shown in Figures 1 (b) and (c) respectively. The energy response of each layer was then calculated, as labelled in Figure 3(a).

Single HD-V2 and EBT2 layers were calibrated against known doses at the Birmingham cyclotron (10 mm diameter proton beam of energy $29 \mathrm{MeV} \pm 1 \%$ [29]) as shown in Figure 2. The RCFs were scanned with a standard optical scanner (Epson Perfection V750 Pro) in transmission mode providing 48 bit images comprising of three 16 bit colour channels, with a resolution of 1200 ppi. A sixth order polynomial fit was obtained for the known dose layers for each channel and a code written in Matlab then converted the RGB value of each pixel in a scanned RCF into dose (in Gy) using the curves shown in Figure 2.

A limitation with RCF is that the signal from different ion species cannot be differentiated, and in the first few layers of the stack, under our experimental conditions, the ion signal will contain contributions from both protons and carbon ions. There is also a diffuse low dose signal from $\mathrm{x}$-rays, gamma rays and high energy electrons which, however, can be identified and subtracted digitally.

Quantitative information on energy-resolved proton and carbon fluence can be obtained using a TPS, but as mentioned above, this diagnostic typically selects only a small sample of the beam ( $\sim 100 \mathrm{nsr})$. However, the often structured nature of the ion beams from ultrathin foil interactions makes a more extensive assessment of the flux and spectrum across the beam particularly relevant. Alternatively to TPS, spectra can also be obtained using RCF, with lower energy resolution but the ability to sample across the entire beam profile [30,31]. A deconvolution procedure is needed to obtain the particle spectrum, as described, for example, in Section III D of Reference [32], as well as in [33] and [34]. 


\section{$2.2 \quad$ CR-39}

CR-39 is a solid state nuclear track detector [35], composed of a plastic polymer $\left(\mathrm{C}_{12} \mathrm{H}_{18} \mathrm{O}_{7}\right)$ with density $1.3 \mathrm{~g} / \mathrm{cm}^{3}$. CR-39 is also used extensively in laser-driven ion acceleration experiments due to its capability of recording directly, the absolute number of ions. Carbon ions and protons create small damage tracks when stopped in CR-39; after irradiation these tracks are revealed and enlarged by a chemical etching process. The surfaces of the etched samples are observed under an optical microscope and the location, size and number of tracks are recorded. CR-39 is insensitive to x-rays and electrons and can distinguish between different ion species (e.g. carbon ions and protons in our case), as for equal etching time, pit size varies for different ions [36-38].

The number of particles on CR-39 can be counted to obtain a particle fluence, distinguishing between carbon and proton ions according to the pit size. By capturing many images across the surface of CR-39, one can obtain a spatial profile of the particle density across the beam. However, in many cases and especially for low energy ions, the surface of the CR-39 can be saturated (typically for fluences $\sim 10^{8}$ particles $/ \mathrm{cm}^{2}$ and above); in this scenario, even after 30 seconds of etching the carbon pits are indistinguishable due to the high particle density. Despite this, qualitative information on the carbon beam (e.g. beam shape, divergence, broad structure) can be obtained from an optical inspection of the CR-39.

CR-39 is etched in a $6.5 \mathrm{~mol} \mathrm{NaOH}$ solution kept at $\sim 85^{\circ} \mathrm{C}$. Etching typically takes less than five minutes and more than ten minutes respectively for carbon and proton pits to be detectable with a $\times 50$ microscope. Overetching can occur in CR-39, where pits overlap and become indistinguishable. The time taken to overetch will depend on the particles' density per unit surface and pit size. Some species may be overetched before other species are detectable [39, 40].

In a stack composed solely of layers of CR-39, the high energy cut-off for protons can be determined by identifying the last layer containing proton pits [41, 42]. Using a multi-step etching technique, the high energy cut-off for both protons and carbons can be identified. By etching the CR-39 pieces for less than 5 minutes, only carbon ions will be visible, so the last layer containing signal can be recorded. After the cut-off of carbon ions is identified, further etching shows proton pits. Typically, protons travel further into the stack and later layers which had no signal from carbon ions will show signal from protons.

\section{Application to Experiments}

\subsection{RCF}

An example of RCF data obtained in this campaign is shown in Figure 3, displaying a profile which varies significantly for different proton energies. At low energy, a ring like structure is visible in the beam profile whereas at higher energies the protons are concentrated in a dense, low divergence component (e.g. Figure 3(b) and (c)). Such a ring structure has been connected to the onset of relativistic transparency under similar interaction conditions [15].

For the stack shown in Figure 3, a cut off proton energy of $20.8 \pm 0.5 \mathrm{MeV}$ can be identified, corresponding to the average of the proton energy having its Bragg peak in the last RCF film with signal (layer 22) and the one corresponding to the following RCF film (layer 23). 


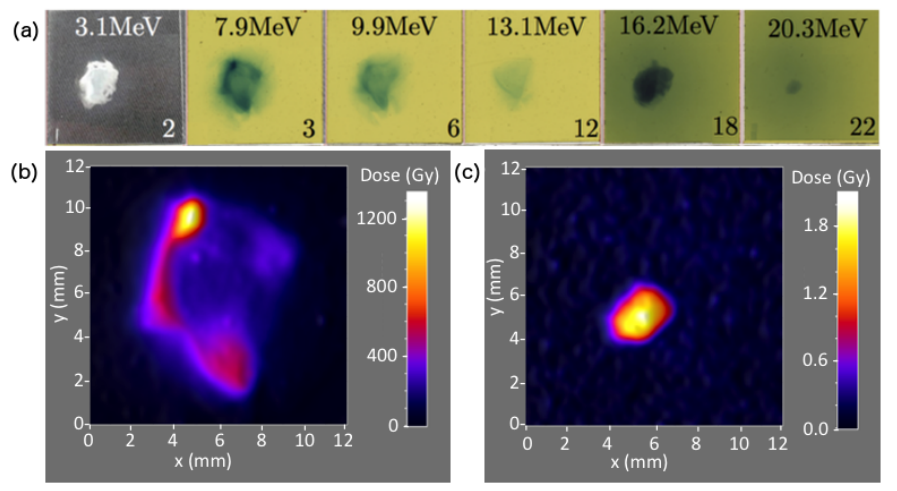

Figure 3. (a) CR-39 and RCF layers from a linear polarization (LP) shot on $10 \mathrm{~nm}$ amorphous carbon target. Layer numbers are denoted on bottom right. Layer 2 is CR-39 (500 $\mu \mathrm{m})$, layers 3, 6 and 12 are HD-V2 and layers 18 and 22 are EBT2. The value at the top of each layer represents the energy of the protons reaching their Bragg peak in the active layer of the film. Dose profiles are shown for layer 3 (b) and layer 22 (c).

In the experimental conditions where the data of Figure 3 was obtained and for the same target thickness and laser polarization, the maximum energy of carbon ions detected on the TPS was < $10 \mathrm{MeV} / \mathrm{u}$, therefore through stopping calculations one could assume reliably that, beyond the 3rd HD-V2 layer, protons (of energy $7.6 \mathrm{MeV}$ and higher) would be the only particles contributing to the ion signal in the stack. For the stack shown in Figure 3, a reliable spectral fluence can therefore be deconvolved for proton energies greater than 7.6 MeV. Figure 4(a) shows the spectra obtained from RCF (selecting an aperture of 2 msr, as marked on Figure 4(b)) compared with a spectrum obtained from the TPS which selects an aperture of $0.1 \mathrm{msr}$ along the laser axis under similar conditions. The spectrum from region I shows a higher fluence for lower energy protons and also a lower maximum energy cut off. The spectrum from region II is quite similar to that obtained from the TPS, as expected, since both are from approximately the same location on similar shots.

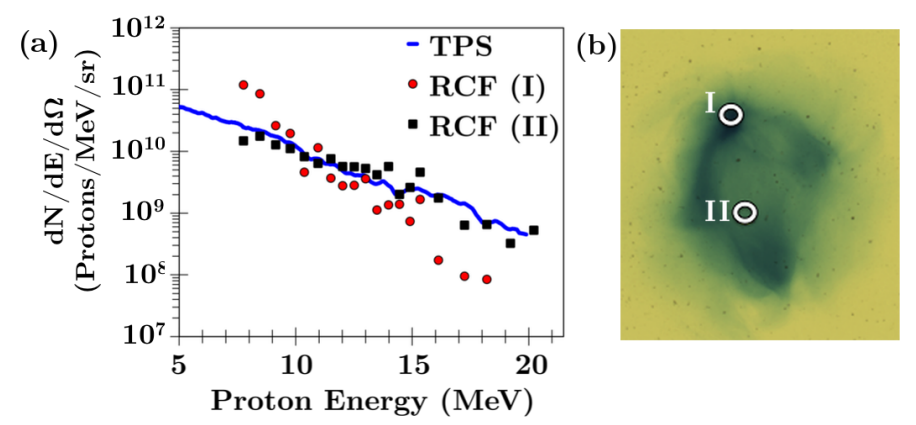

Figure 4. (a) Proton spectra from the RCF stack shown in Fig. 3 and TPS (solid line) for $10 \mathrm{~nm}$ carbon LP shots under similar conditions. (b) The areas I and II marked on the RCF (HD-V2 layer 3) in (b) show where the spectra were taken from through the stack. Region I is aligned with the highest dose on layer 3 . Region II is aligned with the axis of the high energy component of the proton beam (see Fig. 3). 


\subsection{CR-39}

Stacks composed solely of $500 \mu \mathrm{m}$-thick CR-39 (with an initial $9 \mu \mathrm{m}$ Al filter) could determine the high energy cut-off for both protons and carbons. Figure 5 shows CR-39 taken from a shot where more efficient acceleration was achieved than in the shot of Figure 3. It can be seen that there are no carbon ions on the front surface of layer 5 since no pits were detected after 4 minutes of etching. In the shot shown in Figure 5, protons travelled to the 7th layer in the stack. In this particular shot, the cut off energies for carbon ions and protons were determined as $26 \pm 2 \mathrm{MeV} / \mathrm{u}$ and $21.9 \pm 0.8$ $\mathrm{MeV}$ respectively, which is indicative of very efficient volumetric acceleration, a peculiar feature of this interaction regime.

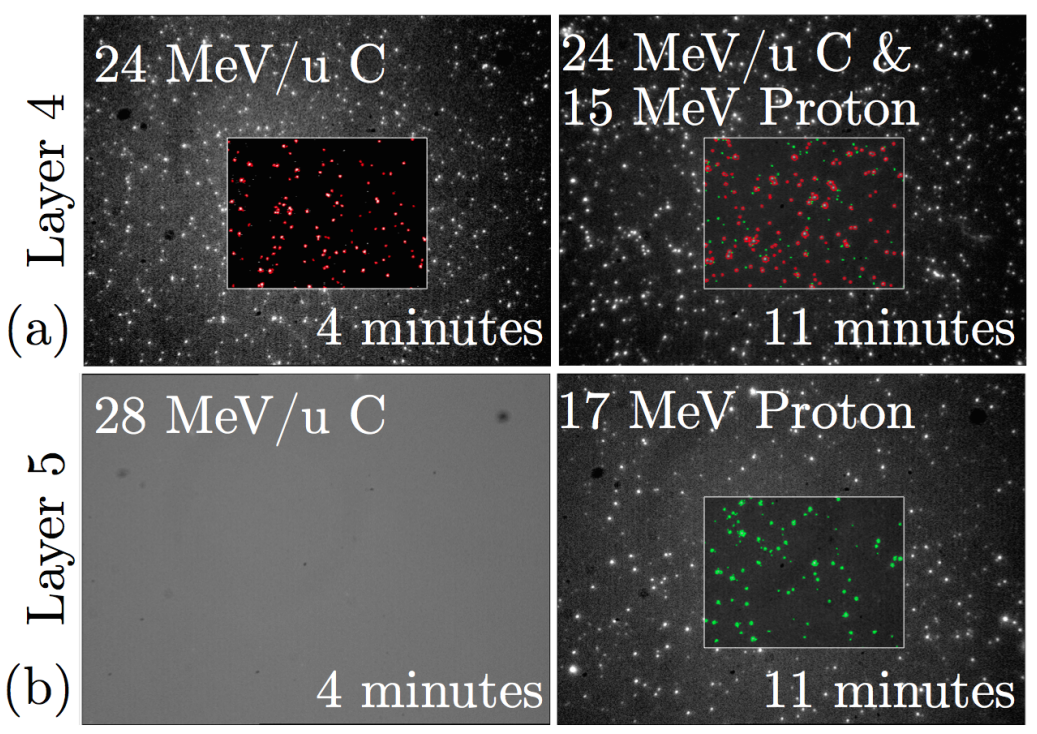

Figure 5. Data from a shot on $10 \mathrm{~nm}$ amorphous carbon target irradiated by a circularly polarized laser pulse. $\times 50$ images of proton (green in inset) and carbon (red in inset) pits from the front surface of two layers of $500 \mu \mathrm{m} \mathrm{CR}-39$ from a stack of CR-39. Layer 4 (a) (15 MeV proton and $24 \mathrm{MeV} / \mathrm{u}$ C) and layer 5 (b) $(17 \mathrm{MeV}$ proton and $28 \mathrm{MeV} / \mathrm{u} \mathrm{C})$ were each etched for 4 (left) and 11 (right) minutes. Only carbon pits are visible after 4 minutes. It is clear that no $28 \mathrm{MeV} / \mathrm{u}$ carbon ions are present.

\subsection{RCF and CR-39 layers}

The capability of providing independent profiles for carbon ions and protons is an important feature of this diagnostic approach, in view of the characterization of volumetric acceleration in this specific regime. Coarse beam profiles can be obtained from CR-39 for carbon species and this can be compared to the profiles obtained from RCF. In cases where the particle flux was too high to obtain quantitative data from CR-39, it was useful to etch for a short period of time $(<3 \mathrm{~min}-$ utes) to obtain a coarse beam profile for low energy carbon ions $(<10 \mathrm{MeV} / \mathrm{u})$ as shown in three shots in Figure 6(a) which have contrasting beam profiles. It was generally observed throughout the experiment that protons and carbon transverse profiles displayed similar broad characteristics at comparable energy per nucleon, as also indicated by particle-in-cell (PIC) simulations of the acceleration process[12]. 
Under the right conditions it is possible to obtain in the same shots independent quantitative lineouts for carbon ions and protons at comparable energy/nucleon $(\sim 6 \mathrm{MeV} / \mathrm{u}$ for carbon and $\sim 8$ $\mathrm{MeV}$ for protons), from CR-39 (front surface layer 2) and RCF (layer 3) respectively, as shown in Figure 6(c). This was possible since the CR-39 layer, (which captured carbon ions with energies from $5.6-14.4 \mathrm{MeV} / \mathrm{u}$ ) was etched for 4 minutes to reveal carbon ions only and for this particular shot the maximum proton energy was $13.6 \mathrm{MeV}$; with this relatively low maximum proton energy it is unlikely that there were carbon ions with energy greater than $14.4 \mathrm{MeV} / \mathrm{u}$ so we can safely assume that the carbon ions stopped in the CR-39 layer and did not contribute to the signal in RCF layer 3 i.e. Fig 6(b). The ringed structure in the low energy carbon profile is similar to that shown in the first RCF layers in Figure 3 which may be again indicative of a relativistic transparency regime. With appropriate positioning of CR-39 in the stack and suitable choice of the CR-39 thickness, this capability of obtaining simultaneous profiles of carbon ions and protons at comparable energies can be extended to higher energies.
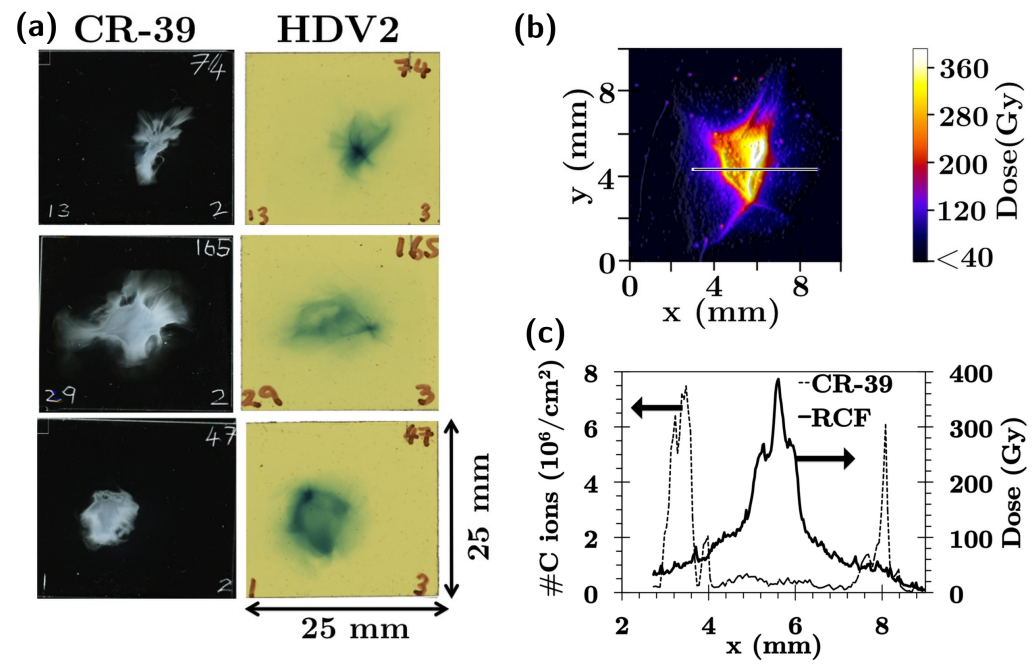

Figure 6. (a) Coarse beam profiles for saturated CR-39 layer 2 (carbon beam profile etched for less than 5 minutes) and HD-V2 layer 3 (generally containing protons and carbons) from $2.5 \mathrm{~nm}$ (top), $5 \mathrm{~nm}$ (middle) and $10 \mathrm{~nm}$ (bottom) amorphous carbon targets and LP laser pulses. (b) Dose profile at $\sim 8 \mathrm{MeV}$ for a $10 \mathrm{~nm}$ LP shot. (c) CR-39 density for carbon ions $(\sim 6 \mathrm{MeV} / \mathrm{u})$ and proton dose profile for the same spatial lineout marked in (b).

\section{Conclusions}

Interactions of high intensity, ultrashort laser pulses with ultrathin ( $\mathrm{nm}$ scale) foils are characterized by efficient acceleration not only of protons, but also of higher-Z ions (e.g. $\mathrm{C}^{6+}$ when employing carbon foils), which can reach similar energies per nucleon. The beam profile is typically structured, unlike the smoother beams obtained from TNSA from thicker foils, and the characteristics of the structure are often particular to an acceleration process [1] and can therefore provide important information. In this paper we have discussed the implementation of approaches which provide spatially/angularly resolved information on the beam profile, including spectral information which 
complements and support TPS diagnostics. While these methods are well established, applying them in this specific context requires some additional consideration and care in order to obtain meaningful information.

\section{Acknowledgments}

We acknowledge the support of the staff at the Central Laser Facility, Rutherford Appleton Laboratory. This work was supported by EPSRC (grants n. EP/K022415/1, EP/J500094/1, EP/L002221/1, EP/J003832/1, EP/J002550/1) STFC (grant ST/J002062/1) and LASERLAB Europe (EC GA 284464).

Data associated with research published in this paper can be accessed at http://dx. doi.org/10.17034/11e2387c7d3c-4e42-8530-314a1eb26eda.

\section{References}

[1] A. Macchi et al., Rev. Mod. Phys., 85, 751 (2013).

[2] T. Tajima et al. Rev. Accel. Sci. Technol., 02, 201 (2009).

[3] B.M. Hegelich et al. Nucl. Fusion, 51, 083011 (2011).

[4] R.A. Snavely et al. Phys. Rev. Lett., 85, 2945 (2000).

[5] S.C. Wilks et al., Phys. Plasmas, 8, 542 (2001).

[6] I.J. Kim et al., Phys. Rev. Lett., 111, 165003 (2013).

[7] J.H. Bin et al., Phys. Rev. Lett.,115, 064801 (2015).

[8] A. Henig et al., Phys. Rev. Lett., 103, 245003 (2009).

[9] D. Gwynne et al., Rev. Sci. Instrum., 85, 033304 (2014).

[10] D. Doria et al., Rev. Sci. Instrum., 86, 123302 (2015).

[11] T. Jeong et al., Rev. Sci. Instrum., 87, 083301 (2016).

[12] A. Sgattoni et al., Phys. Rev. E, 91, 013106 (2015).

[13] C.A.J. Palmer et al., Phys. Rev. Lett., 108, 225002 (2012).

[14] S. Kar et al., Phys. Rev. Lett., 109, 185006 (2012).

[15] R.J. Gray et al., New J. Phys., 16, 093027 (2014).

[16] D. Jung et al., New J. Phys., 15, 123035 (2013).

[17] F. Wagner et al., Phys. Plasmas, 22, 063110 (2015).

[18] H.W. Powell et al., New J. Phys., 17, 103033 (2015).

[19] B. Dromey et al., Rev. Sci. Instrum., 75, 645 (2004).

[20] A Niroomand-Rad et al., Med. Phys., 25, 2093 (1998).

[21] P.R. Bolton et al., Physica Medica, 30, 255270 (2014).

[22] D. Kirby et al., Lasers Part. Beams, 29, 231(2011).

[23] M. Seimetz et al., IEEE Trans. Nucl. Sci., 62, 3216 (2015).

[24] J. Kaufman et al., Proc. SPIE, 9515, 9515J (2015). 
[25] http://www.ashland.com/Ashland/Static/Documents/ ASI/Advanced\%20Materials/gafchromic-hdv2.pdf

[26] http://www.ashland.com/Ashland/Static/Documents/ ASI/Advanced\%20Materials/ebt2.pdf

[27] S. Reinhardt et al., Radiat. Environ. Biophys., 54, 71 (2015).

[28] http://www.srim.org/

[29] D. Kirby et al., Phys. Med. Biol., 55, 417433 (2010).

[30] E. Breschi et al., Laser Part. Beams, 22, 393 (2004).

[31] D. Hey et al., Rev. Sci. Instrum., 79, 053501 (2008).

[32] F. Nurnberg et al., Rev. Sci. Instrum., 80, 033301 (2009).

[33] E. Breschi et al., Nucl. Instr. Meth. Phys. Res., 522, 190 (2004).

[34] M. Schollmeier et al., Rev. Sci. Instrum., 85, 043305 (2014).

[35] R. L. Fleischer et al., J. Appl. Phys., 36, 3645 (1965).

[36] N. Sinenian et al., Rev. Sci. Instrum., 82, 103303 (2011).

[37] M. Kanasaki et al., PPCF,, 58, 034013 (2016).

[38] C. Baccou et al., Rev. Sci. Instrum., 86, 083307 (2015).

[39] S. Gaillard et al., Rev. Sci. Instrum., 78, 013304 (2007).

[40] M.J. Rosenberg et al., Rev. Sci. Instrum., 85, 043302 (2014).

[41] E.L. Clark et al., Phys. Rev. Lett., 85, 1654 (2000).

[42] M. Kanasaki et al., Radiation Meas., 50, 46 (2013). 